\title{
Effect of phenyl ring substitution on J-aggregate formation ability of novel bisazomethine dyes in vapour-deposited films
}

Byung-Soon Kim ${ }^{\mathrm{a}}$, Daisuke Kashibuchi ${ }^{\mathrm{b}}$, Young-A Son ${ }^{\mathrm{c}}$, Sung-Hoon Kim ${ }^{\mathrm{d}}$ and Shinya Matsumoto $^{\mathrm{a}, \mathrm{b}^{*}}$

${ }^{a}$ Graduate School of Environmental and Information Sciences, Yokohama National University, Hodogayaku, Yokohama 240-8501, Japan

${ }^{b}$ Faculty of Education and Human Sciences, Yokohama National University, Hodogaya-ku, Yokohama 240-8501, Japan

${ }^{c}$ School of Chemical and Biological Engineering, Chungnam National University, Daejeon 305-764, Korea

${ }^{d}$ Department of Textile System Engineering, Kyungpook National University, Daegu 702-701, Korea

\begin{abstract}
Bisazomethine dyes, which are synthesized using diaminomaleonitrile and aminobenzaldehydes, exhibit red fluorescence in solution and in the solid state. Several bisazomethine dyes are known to form Jaggregates in vapour-deposited films. In this work, novel bisazomethine dyes were synthesized and the effect of phenyl ring substitution on the J-aggregate formation in vapour-deposited films was examined. The optical properties of the dyes were examined in solution and in the solid state through molecular orbital calculations. Four derivatives were found to form J-aggregates in vapour-deposited films as determined from the shape of the spectrum and the absorption edge.
\end{abstract}

Keywords: bisazomethine dye, J-aggregates, vapour-deposited film, optical properties, molecular orbital calculations. 
*Corresponding author. Tel.: +81 $45 \quad 339$ 3366; Fax: +81 45 339 3345. E-mail address: smatsu@edhs.ynu.ac.jp (S. Matsumoto). 


\section{Introduction}

In recent years, organic molecular materials have gained considerable importance from the viewpoint of their applications in optoelectronics: these materials can be used to fabricate optoelectronic devices such as organic light-emitting diodes, organic thin-film transistors, and organic solar cells [1-9]. Devices fabricated using organic molecular materials are expected to be lightweight and flexible; moreover, the fabrication process is environmentally friendly [10-12]. Many studies have focused on the development of novel organic molecular materials for the abovementioned applications [13, 14] with the aim of obtaining devices with high performance characteristics. However, it is difficult to design the solid-state structure of organic molecules with the desired properties. This is because very little is known about the crystallization phenomena of organic molecules.

Molecular aggregates have attracted considerable attention as potential candidates for improving the characteristics of optoelectronic devices, with the most well-known example of molecular aggregates being J-aggregates. J-aggregates are characterized by sharp red-shifted absorption and strong photoluminescence with respect to the corresponding molecular electronic states [15-22]. These characteristic features are attributed to the excitonic interaction within the aggregates [23]. Scheibe [24] and Khun [25] reported plausible low-dimensional molecular arrangements of J-aggregates. Kato experimentally demonstrated that merocyanine J-aggregates have a brick-wall arrangement in a LB-film and that the electronic states of the film can be interpreted on the basis of exciton interaction [17].

In this study, we have synthesized bisazomethine dyes by using diaminomaleonitrile and benzaldehydes. Crystal structure studies on some bisazomethine dyes [26-28] have revealed that this dye system has a potential to form J-aggregates [20, 22, 29]. The spectra of several bisazomethine dyes in vapour-deposited films were remarkably different from those of their solutions; the spectra in the former case showed a sharp absorption band in the long-wavelength region $[30,31]$. For $N, N^{\prime}-$ bis $[4-(N, N$ diethylamino)benzylidene]-diaminomaleonitrile (DE2), this band was assigned to J-aggregates [31]. J- 
aggregate films of DE2, however, also include a non-aggregate solid phase corresponding to the single crystal phase [26]. This phase mixing was thought to be the cause of the weak fluorescence of J-aggregate films of DE2 [32]. In a previous study, we examined the photovoltaic properties of J-aggregate films of DE2; although we did not find an apparent contribution of J-aggregates in the photocurrent action spectra of the films, we found that the films are a potential photocarrier-generating material comparable to metal phthalocyanines [33]. Thus, it may be possible to improve the solid state properties of this dye system by using a pure J-aggregate solid film. This report describes the effect of phenyl ring substitution on Jaggregate formation in vapour-deposited films. The dyes were synthesized by the reaction of diaminomaleonitrile with 4-(dimethylamino)-2-alkoxybenzaldehydes (1a-7a) and 4-(diethylamino)-2alkoxybenzaldehydes (8a-14a). The UV-Vis absorption and fluorescent properties of the synthesized dyes were investigated in solution as well as in vapour-deposited films. The electronic states of the dyes were characterized by molecular orbital calculations.

\section{Experimental}

\subsection{Materials and equipment}

3-(Dimethylamino)phenol (97\%) and 4-(diethylamino)salicylaldehyde (98\%) were purchased from Sigma-Aldrich Co. Alkylbromides (98\%) and diaminomaleonitrile (98\%) were obtained from Wako Pure Chem. Ind., Ltd. and Nacalai Tesque Inc., respectively. Wakogel C-300 (silica gel) was used for the column chromatography. The ${ }^{1} \mathrm{H}$ NMR spectra were recorded on an EX $270 \mathrm{MHz}$ NMR instrument (Jeol Ltd.) and a DRX $300 \mathrm{MHz}$ NMR instrument (Bruker Co.) with TMS as the internal standard. The elemental analyses were recorded on a Vario EL III elemental analyzer (Elementar Co.). The IR spectra were recorded using a PerkinElmer Spectrum BX FTIR system spectrophotometer. The absorption spectra 
of the bisazomethine dyes and those of their vapour-deposited films were determined using a PerkinElmer Lambda 20 UV-Vis spectrophotometer and a PerkinElmer Lambda 750 UV-Vis spectrophotometer, respectively. Fluorescence spectra measurements were performed on a LS 45 spectrofluorimeter (PerkinElmer) equipped with a xenon discharge lamp and 1-cm quartz cells. The fluorescence spectra of the vapour-deposited films were recorded using a LS 55 spectrofluorimeter (PerkinElmer). The concentration of the solutions used for absorption and fluorescence measurements was ca. $1.0 \times 10^{-5} \mathrm{M}$. Thermal analysis of the synthesized dyes was carried out by using a MEL-TEMP electrothermal melting point apparatus without corrections. Thin films of the synthesized novel bisazomethine dyes were vapourdeposited onto a glass substrate using vacuum deposition equipment (ULVAC VPC-060) under vacuum conditions of ca. $3.0 \times 10^{-2} \mathrm{~Pa}$. All measurements were taken at room temperature at approximately $298 \mathrm{~K}$.

\subsection{Molecular orbital calculations}

The molecular structure for the calculation of the electronic states of a molecule was optimized by using the AM1 Hamiltonian [34]. Theoretical absorption properties were obtained by the INDO/S Hamiltonian $[35,36]$ on the basis of the optimized molecular structure. 257 configuration interactions were taken into account. All the calculations were performed with a CAChe 5.2 program package [37].

\subsection{Synthesis and characterization}

Novel bisazomethine dyes were synthesized according to a previously described method [38, 39], and their chemical structure was confirmed by ${ }^{1} \mathrm{H}$ NMR, IR, and elemental analysis. The synthetic procedure is illustrated in Scheme 1. 
2.3.1 4-(Dimethylamino)salicylaldehyde, 4-(dimethylamino)-2-alkoxybenzaldehydes 1a-7a and 4(diethylamino)-2-alkoxybenzaldehydes 8a-14a

4-(Dimethylamino)salicylaldehyde was synthesized by the reported procedure [40]. To the mixture of 3-(dimethylamino)phenol $20 \mathrm{mmol}(2.75 \mathrm{~g})$ and $10 \mathrm{ml} \mathrm{N}, \mathrm{N}$-dimethylformamide was dropwise added 42 mmol phosphorus oxychloride $(3.46 \mathrm{~g})$ at $0{ }^{\circ} \mathrm{C}$ while stirring. The resulting mixture was stirred at $80{ }^{\circ} \mathrm{C}$ for $16 \mathrm{~h}$. After $16 \mathrm{~h}$, the reaction was cooled to room temperature and the mixture was quenched into 150 $\mathrm{ml}$ ice water. The formed precipitate solution was adjusted to the neutral $\mathrm{pH}$ condition using $4 \mathrm{M}$ sodium hydroxide solution. The solid was filtered and purified by column chromatography by eluting with ethyl acetate $n$-hexane (ethyl acetate: $n$-hexane $=1: 3 \mathrm{v} / \mathrm{v}$ ). The yellow solid was obtained after recrystalization with $n$-hexane. Yield: $61.2 \%$ (2.02 g); M.p. $78-79{ }^{\circ}$ C. ${ }^{1} \mathrm{H}$ NMR $\left(300 \mathrm{MHZ}, \mathrm{CDCl}_{3}\right): \delta 10.73(\mathrm{~s}, 1 \mathrm{H}, \mathrm{CHO})$, $\delta 8.64(\mathrm{~s}, 1 \mathrm{H}, \mathrm{OH}), \delta 6.38 \sim 6.43(\mathrm{t}, J=8.8 \mathrm{~Hz}, 1 \mathrm{H}$, aromatic), $\delta 5.40 \sim 5.44(\mathrm{dd}, J=6.6$ and $2.3 \mathrm{~Hz}, 1 \mathrm{H}$, aromatic), $\delta 5.21 \sim 4.22\left(\mathrm{~d}, J=2.3 \mathrm{~Hz}, 1 \mathrm{H}\right.$, aromatic), $\delta 2.17 \sim 2.19\left(\mathrm{~d}, J=5.0 \mathrm{~Hz}, 6 \mathrm{H}, \mathrm{NMe}_{2}\right)$; IR $(\mathrm{KBr})$ : $1646(\mathrm{C}=\mathrm{O}) \mathrm{cm}^{-1}$.

A mixture of $10 \quad \mathrm{mmol} \quad$ 4-(dimethylamino)salicylaldehyde $\quad\left(\begin{array}{lllll}1.66 & \mathrm{~g}) & \text { (or } & \text { 4- }\end{array}\right.$ (diethylamino)salicylaldehyde (1.94 g)) and $20 \mathrm{mmol}$ potassium carbonate $(2.76 \mathrm{~g})$ was dissolved in 30 $\mathrm{ml}$ dry DMF. To this mixture was added $30 \mathrm{mmol}$ alkyl bromide and the mixture was refluxed for $8 \mathrm{~h}$ at $50{ }^{\circ} \mathrm{C}$. The reaction mixture was cooled to room temperature and filtered to remove potassium carbonate. The mixture was then poured into ice water and extracted with ethyl acetate. The combined organic layer was washed with water and brine and dried over magnesium sulphate. The solvent was evaporated under reduced pressure and then purified by column chromatography by eluting with ethyl acetate/n-hexane (ethyl acetate: $n$-hexane $=1: 3 \mathrm{v} / \mathrm{v}$ ) and recrystallization with $n$-hexane. These bright brown compounds 1a-14a were found to melt around $30-32{ }^{\circ} \mathrm{C}$. To obtain 2a and 9a, $10 \mathrm{mmol}$ of copper (I) oxide (1.43 g) was added as a catalyst and the reaction conditions were changed to $5 \mathrm{~h}$ and $100^{\circ} \mathrm{C}$. 1a [41-43], Yield: 
$87.6 \%(2.24 \mathrm{~g}) ;{ }^{1} \mathrm{H}$ NMR $\left(300 \mathrm{MHz}, \mathrm{CDCl}_{3}\right): \delta 10.21(\mathrm{~s}, 1 \mathrm{H}, \mathrm{CHO}), \delta 7.68 \sim 7.71(\mathrm{~d}, J=8.8 \mathrm{~Hz}, 1 \mathrm{H}$, aromatic), $\delta 7.27 \sim 7.40(\mathrm{~m}, 5 \mathrm{H}, \mathrm{O}-\mathrm{Bz}), \delta 6.29(\mathrm{~d}, J=1.5 \mathrm{~Hz}, 1 \mathrm{H}$, aromatic), $\delta 6.26(\mathrm{~d}, J=1.5 \mathrm{~Hz}, 1 \mathrm{H}$, aromatic), $\delta 5.11(\mathrm{~s}, 2 \mathrm{H}, \mathrm{O}-\mathrm{Bz}), \delta 2.99\left(\mathrm{~s}, 6 \mathrm{H}, \mathrm{NMe}_{2}\right)$; IR $(\mathrm{KBr}): 1651(\mathrm{C}=\mathrm{O}) \mathrm{cm}^{-1} .2 \mathrm{a}$ [44-46], Yield: $33.2 \%(0.80 \mathrm{~g}) ;{ }^{1} \mathrm{H}$ NMR $\left(300 \mathrm{MHz}, \mathrm{CDCl}_{3}\right): \delta 10.10 \sim 10.11$ (s, $\left.1 \mathrm{H}, \mathrm{CHO}\right), \delta 7.76 \sim 7.79(\mathrm{~d}, J=8.8 \mathrm{~Hz}, 1 \mathrm{H}$, aromatic), $\delta 7.27 \sim 7.34$ (m, 2H, O-Ph), $\delta 6.97 \sim 7.09$ (m, 3H, O-Ph), $\delta 6.43 \sim 6.47$ (dd, $J=6.4$ and $2.4 \mathrm{~Hz}, 1 \mathrm{H}$, aromatic), $\delta 5.99 \sim 6.00\left(\mathrm{~d}, J=2.5 \mathrm{~Hz}, 1 \mathrm{H}\right.$, aromatic), $\delta 2.92\left(\mathrm{~s}, 6 \mathrm{H}, \mathrm{NMe}_{2}\right) ; \mathrm{IR}(\mathrm{KBr}): 1664(\mathrm{C}=\mathrm{O}) \mathrm{cm}^{-1} .3 \mathrm{a}$, Yield: $68.1 \%$ (1.22 g); ${ }^{1} \mathrm{H}$ NMR (270 MHz, $\left.\mathrm{CDCl}_{3}\right): \delta 10.15$ (s, $\left.1 \mathrm{H}, \mathrm{CHO}\right), \delta 7.69 \sim 72(\mathrm{~d}, J=8.1 \mathrm{~Hz}, 1 \mathrm{H}$, aromatic), $\delta 6.27 \sim 6.31$ (dd, $J=8.1$ and $2.7 \mathrm{~Hz}, 1 \mathrm{H}$, aromatic), $\delta 6.01 \sim 6.02$ (s, $1 \mathrm{H}$, aromatic), $\delta 3.89$ (s, $3 \mathrm{H}$, O-Me), $\delta 3.07\left(\mathrm{~s}, 6 \mathrm{H}, \mathrm{NMe}_{2}\right)$; IR (KBr): $1656(\mathrm{C}=\mathrm{O}) \mathrm{cm}^{-1} .4 \mathrm{a}$, Yield: 52.6\% $(1.09 \mathrm{~g}) ;{ }^{1} \mathrm{H}$ NMR $(270 \mathrm{MHz}$, $\left.\mathrm{CDCl}_{3}\right): \delta 10.23(\mathrm{~s}, 1 \mathrm{H}, \mathrm{CHO}), \delta 7.72 \sim 75(\mathrm{~d}, J=8.1 \mathrm{~Hz}, 1 \mathrm{H}$, aromatic), $\delta 6.28 \sim 6.32(\mathrm{dd}, J=8.1$ and $2.7 \mathrm{~Hz}$, $1 \mathrm{H}$, aromatic), $\delta 6.03 \sim 6.04$ (d, $J=2.7 \mathrm{~Hz}, 1 \mathrm{H}$, aromatic), $\delta 3.99 \sim 4.03$ (t, $J=5.4 \mathrm{~Hz}, 2 \mathrm{H}, \mathrm{O}-\mathrm{Pr}$ ), $\delta 3.07$ (s, 6H, $\mathrm{NMe}_{2}$ ), $\delta 1.80 \sim 1.93$ (m, 2H, O-Pr), $\delta 1.04 \sim 1.10$ (t, $\left.J=5.4 \mathrm{~Hz}, 3 \mathrm{H}, \mathrm{O}-\mathrm{Pr}\right)$; IR (KBr): $1660(\mathrm{C}=\mathrm{O}) \mathrm{cm}^{-1}$. 5a, Yield: 64.9\% (1.44 g); ${ }^{1} \mathrm{H}$ NMR (270 MHz, $\left.\mathrm{CDCl}_{3}\right): \delta 10.21$ (s, $\left.1 \mathrm{H}, \mathrm{CHO}\right), \delta 7.70 \sim 7.74(\mathrm{~d}, J=8.9 \mathrm{~Hz}$, $1 \mathrm{H}$, aromatic), $\delta 6.26 \sim 6.30$ (m, 1H, aromatic), $\delta 6.02 \sim 6.03$ (d, $J=2.1 \mathrm{~Hz}, 1 \mathrm{H}$, aromatic), $\delta 4.02 \sim 4.07$ (t, $J=$ $6.2 \mathrm{~Hz}, 2 \mathrm{H}, \mathrm{O}-\mathrm{Bu}), \delta 3.06\left(\mathrm{~s}, 6 \mathrm{H}, \mathrm{NMe}_{2}\right), \delta 1.77 \sim 1.87(\mathrm{~m}, 2 \mathrm{H}, \mathrm{O}-\mathrm{Bu}), \delta 1.45 \sim 1.59(\mathrm{~m}, 2 \mathrm{H}, \mathrm{O}-\mathrm{Bu})$, $\delta 0.96 \sim 1.01(\mathrm{t}, J=7.3 \mathrm{~Hz}, 3 \mathrm{H}, \mathrm{O}-\mathrm{Bu})$; IR (KBr): $1660(\mathrm{C}=\mathrm{O}) \mathrm{cm}^{-1}$. 6a, Yield: 67.6\% (1.59 g); ${ }^{1} \mathrm{H}$ NMR (300 MHz, DMSO-d $\left.{ }_{6}\right): \delta 10.05$ (s, $\left.1 \mathrm{H}, \mathrm{CHO}\right), \delta 7.49 \sim 7.52$ (d, $J=8.8 \mathrm{~Hz}, 1 \mathrm{H}$, aromatic), $\delta 6.34 \sim 6.37$ (dd, $J$ $=9.0$ and $1.5 \mathrm{~Hz}, 1 \mathrm{H}$, aromatic), $\delta 6.17 \sim 6.18(\mathrm{~d}, J=2.2 \mathrm{~Hz}, 1 \mathrm{H}$, aromatic), $\delta 4.06 \sim 4.11(\mathrm{t}, J=6.4 \mathrm{~Hz}, 2 \mathrm{H}$, O-Pen), $\delta 3.04$ (s, 6H, $\mathrm{NMe}_{2}$ ), $\delta 1.74 \sim 1.79$ (m, 2H, O-Pen), $\delta 1.34 \sim 1.39$ (m, 4H, O-Pen), $\delta 0.87 \sim 0.92$ (t, $J=$ 7.0 Hz, 3H, O-Pen); IR (KBr): $1661(\mathrm{C}=\mathrm{O}) \mathrm{cm}^{-1} .7 \mathrm{a}$, Yield: 56.1\% (1.71 g); ${ }^{1} \mathrm{H}$ NMR (300 MHz, DMSO$\left.\mathrm{d}_{6}\right): \delta 10.05(\mathrm{~s}, 1 \mathrm{H}, \mathrm{CHO}), \delta 7.49 \sim 7.52(\mathrm{~d}, J=8.8 \mathrm{~Hz}, 1 \mathrm{H}$, aromatic), $\delta 6.33 \sim 6.36(\mathrm{~d}, J=8.8 \mathrm{~Hz}, 1 \mathrm{H}$, aromatic), $\delta 6.16 \sim 6.17$ (d, $J=2.3 \mathrm{~Hz}, 1 \mathrm{H}$, aromatic), $\delta 4.06 \sim 4.10$ (t, $J=6.4 \mathrm{~Hz}, 2 \mathrm{H}, \mathrm{O}-\mathrm{Dec}), \delta 3.04$ (s, $6 \mathrm{H}$, $\mathrm{NMe}_{2}$ ), $\delta 1.72 \sim 1.77$ (m, 2H, O-Dec), $\delta 1.24 \sim 1.43$ (m, 14H, O-Dec), $\delta 0.82 \sim 0.86$ (t, $J=6.4 \mathrm{~Hz}, 3 \mathrm{H}, \mathrm{O}-\mathrm{Dec}$ ); IR (KBr): $1664(\mathrm{C}=\mathrm{O}) \mathrm{cm}^{-1}$. 8a, Yield: $35.0 \%(0.99 \mathrm{~g}) ;{ }^{1} \mathrm{H}$ NMR $\left(270 \mathrm{MHz}, \mathrm{CDCl}_{3}\right): \delta 10.23(\mathrm{~s}, 1 \mathrm{H}$, 
CHO), $\delta 7.71 \sim 7.74(\mathrm{~d}, J=8.8 \mathrm{~Hz}, 1 \mathrm{H}$, aromatic), $\delta 7.24 \sim 7.45(\mathrm{~m}, 5 \mathrm{H}, \mathrm{O}-\mathrm{Bz}), \delta 6.12 \sim 6.32(\mathrm{~d}, J=8.2 \mathrm{~Hz}$, 2H, aromatic), $\delta 5.16(\mathrm{~s}, 2 \mathrm{H}, \mathrm{O}-\mathrm{Bz}), \delta 3.31 \sim 3.39$ (q, $J=7.2 \mathrm{~Hz}, 4 \mathrm{H}, \mathrm{NEt}_{2}$ ), $\delta 1.06 \sim 1.23\left(\mathrm{~m}, 6 \mathrm{H}, \mathrm{NEt}_{2}\right.$ ); IR (KBr): $1644(\mathrm{C}=\mathrm{O}) \mathrm{cm}^{-1}$. 9a, Yield: 30.0\% (0.81 g); ${ }^{1} \mathrm{H}$ NMR $\left(300 \mathrm{MHz}, \mathrm{CDCl}_{3}\right): \delta 10.13(\mathrm{~s}, 1 \mathrm{H}, \mathrm{CHO})$, $\delta 7.80 \sim 7.83(\mathrm{~d}, J=8.8 \mathrm{~Hz}, 1 \mathrm{H}$, aromatic), $\delta 7.33 \sim 7.38(\mathrm{~m}, 2 \mathrm{H}, \mathrm{O}-\mathrm{Ph}), \delta 7.03 \sim 7.14(\mathrm{~m}, 3 \mathrm{H}, \mathrm{O}-\mathrm{Ph})$, $\delta 6.47 \sim 6.50(\mathrm{~d}, J=9.0 \mathrm{~Hz}, 1 \mathrm{H}$, aromatic), $\delta 6.02 \sim 6.03(\mathrm{~d}, J=2.0 \mathrm{~Hz}, 1 \mathrm{H}$, aromatic), $\delta 3.28 \sim 3.35$ (q, $J=7.1$ $\left.\mathrm{Hz}, 4 \mathrm{H}, \mathrm{NEt}_{2}\right), \delta 1.10 \sim 1.14\left(\mathrm{t}, J=6.9 \mathrm{~Hz}, 6 \mathrm{H}, \mathrm{NEt}_{2}\right)$; IR $(\mathrm{KBr}): 1658(\mathrm{C}=\mathrm{O}) \mathrm{cm}^{-1} .10 \mathrm{a}$, Yield: $84.8 \%(1.76$ g); ${ }^{1} \mathrm{H}$ NMR (300 MHz, $\left.\mathrm{CDCl}_{3}\right): \delta 10.11 \sim 10.12$ (s, 1H, CHO), $\delta 7.68 \sim 7.71$ (d, $J=8.8 \mathrm{~Hz}, 1 \mathrm{H}$, aromatic), $\delta 6.26 \sim 6.29(\mathrm{dd}, J=7.1$ and $1.6 \mathrm{~Hz}, 1 \mathrm{H}$, aromatic), $\delta 6.02 \sim 6.03(\mathrm{~d}, J=2.4 \mathrm{~Hz}, 1 \mathrm{H}$, aromatic), $\delta 3.88(\mathrm{~s}, 3 \mathrm{H}$, O-Me), $83.39 \sim 3.46$ (q, $J=7.1 \mathrm{~Hz}, 4 \mathrm{H}, \mathrm{NEt}_{2}$ ), $\delta 1.15 \sim 1.24\left(\mathrm{t}, J=7.0 \mathrm{~Hz}, 6 \mathrm{H}, \mathrm{NEt}_{2}\right)$; IR $(\mathrm{KBr}): 1645$ $(\mathrm{C}=\mathrm{O}) \mathrm{cm}^{-1}$. 11a, Yield: 84.9\% (1.88 g); ${ }^{1} \mathrm{H}$ NMR (270 MHz, $\left.\mathrm{CDCl}_{3}\right): \delta 10.17(\mathrm{~s}, 1 \mathrm{H}, \mathrm{CHO}), \delta 7.69 \sim 7.72$ (d, $J=8.9 \mathrm{~Hz}, 1 \mathrm{H}$, aromatic), $\delta 6.25 \sim 6.28(\mathrm{~m}, 1 \mathrm{H}$, aromatic), $\delta 6.01 \sim 6.02(\mathrm{~d}, J=2.4 \mathrm{~Hz}, 1 \mathrm{H}$, aromatic), $\delta 4.06 \sim 4.14$ (q, $J=7.0 \mathrm{~Hz}, 2 \mathrm{H}, \mathrm{O}-\mathrm{Et}), \delta 3.37 \sim 3.45$ (q, $\left.J=7.0 \mathrm{~Hz}, 4 \mathrm{H}, \mathrm{NEt}_{2}\right), \delta 1.43 \sim 1.48(\mathrm{t}, J=7.0 \mathrm{~Hz}, 3 \mathrm{H}$, O-Et), $\delta 1.18 \sim 1.23\left(\mathrm{t}, J=7.0 \mathrm{~Hz}, 6 \mathrm{H}, \mathrm{NEt}_{2}\right)$; IR $(\mathrm{KBr}): 1652(\mathrm{C}=\mathrm{O}) \mathrm{cm}^{-1} .12 \mathrm{a}$, Yield: $77.3 \%(1.82 \mathrm{~g}) ;{ }^{1} \mathrm{H}$ NMR (300 MHz, $\left.\mathrm{CDCl}_{3}\right): \delta 10.19(\mathrm{~s}, 1 \mathrm{H}, \mathrm{CHO}), \delta 7.70 \sim 7.73(\mathrm{~d}, J=9.0 \mathrm{~Hz}, 1 \mathrm{H}$, aromatic), $\delta 6.25 \sim 6.29$ (dd, $J=7.3$ and $1.7 \mathrm{~Hz}, 1 \mathrm{H}$, aromatic), $\delta 6.01$ (d, $J=2.2 \mathrm{~Hz}, 1 \mathrm{H}$, aromatic), $\delta 3.97 \sim 4.01$ (t, $J=6.4 \mathrm{~Hz}, 2 \mathrm{H}, \mathrm{O}-$ Pr), $\delta 3.38 \sim 3.45\left(\mathrm{q}, J=7.1 \mathrm{~Hz}, 4 \mathrm{H}, \mathrm{NEt}_{2}\right), \delta 1.80 \sim 1.92(\mathrm{~m}, 2 \mathrm{H}, \mathrm{O}-\mathrm{Po}), \delta 1.19 \sim 1.24(\mathrm{t}, J=7.1 \mathrm{~Hz}, 6 \mathrm{H}$, $\left.\mathrm{NEt}_{2}\right), \delta 1.10 \sim 1.14(\mathrm{t}, J=7.4 \mathrm{~Hz}, 3 \mathrm{H}, \mathrm{O}-\mathrm{Pr})$; IR $(\mathrm{KBr}): 1655(\mathrm{C}=\mathrm{O}) \mathrm{cm}^{-1}$. 13a, Yield: $72.9 \%(1.92 \mathrm{~g}) ;{ }^{1} \mathrm{H}$ NMR (270 MHz, $\left.\mathrm{CDCl}_{3}\right): \delta 10.19(\mathrm{~s}, 1 \mathrm{H}, \mathrm{CHO}), \delta 7.69 \sim 7.72(\mathrm{~d}, J=8.1 \mathrm{~Hz}, 1 \mathrm{H}$, aromatic), $\delta 6.24 \sim 6.28(\mathrm{dd}$, $J=5.4$ and $2.7 \mathrm{~Hz}, 1 \mathrm{H}$, aromatic), $\delta 6.01 \sim 6.02(\mathrm{~d}, J=2.7 \mathrm{~Hz}, 1 \mathrm{H}$, aromatic), $\delta 4.00 \sim 4.05(\mathrm{t}, J=6.7 \mathrm{~Hz}, 2 \mathrm{H}$, O-Pen), $\delta 3.37 \sim 3.45$ (q, $J=8.1 \mathrm{~Hz}, 4 \mathrm{H}, \mathrm{NEt}_{2}$ ), $\delta 1.78 \sim 1.88$ (m, 2H, O-Pen), $\delta 1.38 \sim 1.51$ (m, 4H, O-Pen), $\delta 1.19 \sim 1.24\left(\mathrm{t}, J=6.7 \mathrm{~Hz}, 6 \mathrm{H}, \mathrm{NEt}_{2}\right), \delta 0.91 \sim 0.95(\mathrm{t}, J=6.7 \mathrm{~Hz}, 3 \mathrm{H}, \mathrm{O}-\mathrm{Pen})$; IR (KBr): $1661(\mathrm{C}=\mathrm{O}) \mathrm{cm}^{-1}$. 14a, Yield: $55.7 \%(1.86 \mathrm{~g}) ;{ }^{1} \mathrm{H}$ NMR $\left(270 \mathrm{MHz}, \mathrm{CDCl}_{3}\right): \delta 10.18(\mathrm{~s}, 1 \mathrm{H}, \mathrm{CHO}), \delta 7.70 \sim 7.73(\mathrm{~d}, J=8.1 \mathrm{~Hz}$, $1 \mathrm{H}$, aromatic), $\delta 6.25 \sim 6.29(\mathrm{~m}, 1 \mathrm{H}$, aromatic), $\delta 6.01 \sim 6.02(\mathrm{~d}, J=2.7 \mathrm{~Hz}, 1 \mathrm{H}$, aromatic), $\delta 4.00 \sim 4.05(\mathrm{t}, J=$ $6.7 \mathrm{~Hz}, 2 \mathrm{H}, \mathrm{O}-\mathrm{Dec}), \delta 3.38 \sim 3.46\left(\mathrm{q}, J=8.1 \mathrm{~Hz}, 4 \mathrm{H}, \mathrm{NEt}_{2}\right), \delta 1.78 \sim 1.88(\mathrm{~m}, 2 \mathrm{H}, \mathrm{O}-\mathrm{Dec}), 1.11 \sim 1.51(\mathrm{~m}$, 
20H, O-Den and NEt 2 ), $\delta 0.86 \sim 0.91$ (t, $J=5.4 \mathrm{~Hz}, 3 \mathrm{H}, \mathrm{O}-\mathrm{Dec})$; IR (KBr): $1661(\mathrm{C}=\mathrm{O}) \mathrm{cm}^{-1}$.

\subsubsection{Bisazomethine dyes $1 b-14 b$}

To $15 \mathrm{ml}$ of benzene was added a mixture of $0.5 \mathrm{mmol}$ diaminomaleonitrile $(0.06 \mathrm{~g})$ and $1.0 \mathrm{mmol}$ of the prepared 4-(dimethylamino)-2-alkoxybenzaldehydes (or 4-(diethylamino)-2-alkoxybenzaldehydes); the resulting mixture was stirred. Piperidine was added dropwise during the reaction. Reflux was continued for $8 \mathrm{~h}$ with a Dean-Stark trap. After $8 \mathrm{~h}$, the reaction mixture was cooled to room temperature. The precipitated crude product was filtered and washed with methanol. The product was then purified by column chromatography using chloroform. The purple solids were obtained after recrytallization from chloroform except for dyes $\mathbf{7 b}$ and $\mathbf{1 4 b}$ which gave red solids. 1b, Yield: 62.0\% (0.18 g); M.p. $310-$ $311{ }^{\circ} \mathrm{C}$. Anal. Calcd for $\mathrm{C}_{36} \mathrm{H}_{34} \mathrm{~N}_{6} \mathrm{O}_{2}: \mathrm{C}, 74.20 ; \mathrm{H}, 5.88 ; \mathrm{N}, 14.42$. Found: $\mathrm{C}, 74.12 ; \mathrm{H}, 5.78 ; \mathrm{N}, 14.13 .{ }^{1} \mathrm{H}$ NMR (300 MHz, $\left.\mathrm{CDCl}_{3}\right): \delta 9.15(\mathrm{~s}, 2 \mathrm{H}, \mathrm{CH}=\mathrm{N}), \delta 8.14 \sim 8.17(\mathrm{~d}, J=9.0 \mathrm{~Hz}, 2 \mathrm{H}$, aromatic), $\delta 7.37 \sim 7.48(\mathrm{~m}$, $10 \mathrm{H}, \mathrm{O}-\mathrm{Bz}$ ), $\delta 6.34 \sim 6.38$ (dd, $J=6.4$ and $2.5 \mathrm{~Hz}, 2 \mathrm{H}$, aromatic), $\delta 6.08 \sim 6.09$ (d, $J=2.3 \mathrm{~Hz}, 2 \mathrm{H}$, aromatic), $\delta 5.22(\mathrm{~s}, 4 \mathrm{H}, \mathrm{O}-\mathrm{Bz}), \delta 3.05\left(\mathrm{~s}, 12 \mathrm{H}, \mathrm{NMe}_{2}\right)$; IR $(\mathrm{KBr}): 2208(\mathrm{C} \equiv \mathrm{N}) \mathrm{cm}^{-1}, 1615(\mathrm{CH}=\mathrm{N}) \mathrm{cm}^{-1} .2 \mathbf{b}$, Yield: 64.3\% (0.18 g); M.p. $312-313{ }^{\circ}$ C. Anal. Calcd for $\mathrm{C}_{34} \mathrm{H}_{30} \mathrm{~N}_{6} \mathrm{O}_{2}$ : C, $73.63 ; \mathrm{H}, 5.45 ; \mathrm{N}, 15.15$. Found: C, 72.79; H, 5.39; N, 15.0. ${ }^{1} \mathrm{H}$ NMR (270 MHz, $\left.\mathrm{CDCl}_{3}\right): \delta 9.01 \sim 9.06(\mathrm{~s}, 2 \mathrm{H}, \mathrm{CH}=\mathrm{N}), \delta 8.20 \sim 8.23(\mathrm{~d}, J=9.1$ $\mathrm{Hz}, 2 \mathrm{H}$, aromatic), $\delta 7.32 \sim 7.38$ (m, 4H, O-Ph), $\delta 7.07 \sim 7.16$ (m, 6H, O-Ph), $\delta 6.55 \sim 6.60$ (dd, $J=7.0$ and 2.2 $\mathrm{Hz}, 2 \mathrm{H}$, aromatic), $\delta 6.12 \sim 6.13\left(\mathrm{~d}, J=2.4 \mathrm{~Hz}, 2 \mathrm{H}\right.$, aromatic), $\delta 2.98 \sim 3.08\left(\mathrm{~m}, 12 \mathrm{H}, \mathrm{NMe}_{2}\right) ; \mathrm{IR}(\mathrm{KBr})$ : $2207(\mathrm{C} \equiv \mathrm{N}) \mathrm{cm}^{-1}, 1612(\mathrm{CH}=\mathrm{N}) \mathrm{cm}^{-1} .3 \mathbf{b}$, Yield: $70.9 \%\left(0.15\right.$ g); M.p. $318-320{ }^{\circ} \mathrm{C}$. Anal. Calcd for $\mathrm{C}_{24} \mathrm{H}_{26} \mathrm{~N}_{6} \mathrm{O}_{2}$ : C, 66.96; H, 6.09; N, 19.52. Found: C, 66.89; H, 6.08; N, 19.50. ${ }^{1} \mathrm{H}$ NMR (270 MHz, $\left.\mathrm{CDCl}_{3}\right): \delta 9.03(\mathrm{~s}, 2 \mathrm{H}, \mathrm{CH}=\mathrm{N}), \delta 8.12 \sim 8.15(\mathrm{~d}, J=8.9 \mathrm{~Hz}, 2 \mathrm{H}$, aromatic), $\delta 6.38(\mathrm{dd}, J=7.1$ and $2.1 \mathrm{~Hz}$, $2 \mathrm{H}$, aromatic), $\delta 6.04 \sim 6.05\left(\mathrm{~d}, J=2.0 \mathrm{~Hz}, 2 \mathrm{H}\right.$, aromatic), $\delta 3.90$ (s, 6H, O-Me), $\delta 3.11$ (s, $12 \mathrm{H}, \mathrm{NMe}_{2}$ ); IR (KBr): $2203(\mathrm{C} \equiv \mathrm{N}) \mathrm{cm}^{-1}, 1608(\mathrm{CH}=\mathrm{N}) \mathrm{cm}^{-1} .4 \mathbf{b}$, Yield: 46.5\% (0.11 g); M.p. 320-321 ${ }^{\circ} \mathrm{C}$. Anal. Calcd 
for $\mathrm{C}_{28} \mathrm{H}_{34} \mathrm{~N}_{6} \mathrm{O}_{2}$ : C, 69.11; H, 7.04; N, 17.27. Found: $\mathrm{C}, 68.86 ; \mathrm{H}, 7.03 ; \mathrm{N}, 17.17 .{ }^{1} \mathrm{H}$ NMR $(270 \mathrm{MHz}$, $\left.\mathrm{CDCl}_{3}\right): \delta 9.03(\mathrm{~s}, 2 \mathrm{H}, \mathrm{CH}=\mathrm{N}), \delta 8.12 \sim 8.15(\mathrm{~d}, J=8.1 \mathrm{~Hz}, 2 \mathrm{H}$, aromatic), $\delta 6.38(\mathrm{~s}, 2 \mathrm{H}$, aromatic), $\delta 6.04 \sim 6.05\left(\mathrm{~d}, J=2.7 \mathrm{~Hz}, 2 \mathrm{H}\right.$, aromatic), $\delta 3.97 \sim 4.02(\mathrm{t}, J=6.7 \mathrm{~Hz}, 4 \mathrm{H}, \mathrm{O}-\mathrm{Pr}), \delta 3.11\left(\mathrm{~s}, 12 \mathrm{H}, \mathrm{NMe}_{2}\right)$, $\delta 1.86 \sim 1.93(\mathrm{~m}, 4 \mathrm{H}, \mathrm{O}-\mathrm{Pr}), \delta 1.07 \sim 1.12(\mathrm{t}, J=6.8 \mathrm{~Hz}, 6 \mathrm{H}, \mathrm{O}-\mathrm{Pr}) ; \mathrm{IR}(\mathrm{KBr}): 2202(\mathrm{C} \equiv \mathrm{N}) \mathrm{cm}^{-1}, 1611$ $(\mathrm{CH}=\mathrm{N}) \mathrm{cm}^{-1} .5 \mathbf{b}$, Yield: $60.4 \%\left(0.16\right.$ g); M.p. $300-302{ }^{\circ} \mathrm{C}$. Anal. Calcd for $\mathrm{C}_{30} \mathrm{H}_{38} \mathrm{~N}_{6} \mathrm{O}_{2}: \mathrm{C}, 70.01 ; \mathrm{H}$, 7.44; N, 16.33. Found: C, 70.12; H, 7.48; N, 16.31. ${ }^{1} \mathrm{H}$ NMR (270 MHz, $\left.\mathrm{CDCl}_{3}\right): \delta 9.04(\mathrm{~s}, 2 \mathrm{H}, \mathrm{CH}=\mathrm{N})$, $\delta 8.11 \sim 8.14(\mathrm{~d}, J=8.2 \mathrm{~Hz}, 2 \mathrm{H}$, aromatic), $\delta 6.33 \sim 6.37$ (dd, $J=7.0$ and $2.2 \mathrm{~Hz}, 2 \mathrm{H}$, aromatic), $\delta 6.04 \sim 6.05$ (d, $J=2.4 \mathrm{~Hz}, 2 \mathrm{H}$, aromatic), $\delta 4.03 \sim 4.08(\mathrm{t}, J=6.5 \mathrm{~Hz}, 4 \mathrm{H}, \mathrm{O}-\mathrm{Bu}), \delta 3.09$ (s, 12H, $\left.\mathrm{NMe}_{2}\right), \delta 1.81 \sim 1.91(\mathrm{~m}$, 4H, O-Bu), $\delta 1.48 \sim 1.61$ (m, 4H, O-Bu), $\delta 0.98 \sim 1.03$ (t, $J=7.3 \mathrm{~Hz}, 6 \mathrm{H}, \mathrm{O}-\mathrm{Bu})$; IR (KBr): $2202(\mathrm{C} \equiv \mathrm{N}) \mathrm{cm}^{-1}$, $1613(\mathrm{CH}=\mathrm{N}) \mathrm{cm}^{-1}$. 6b, Yield: $72.5 \%\left(0.20\right.$ g); M.p. $287-289^{\circ} \mathrm{C}$. Anal. Calcd for $\mathrm{C}_{32} \mathrm{H}_{42} \mathrm{~N}_{6} \mathrm{O}_{2}: \mathrm{C}, 70.82$; $\mathrm{H}, 7.80 ; \mathrm{N}, 15.49$. Found: $\mathrm{C}, 70.42 ; \mathrm{H}, 7.70 ; \mathrm{N}, 15.49 .{ }^{1} \mathrm{H} \mathrm{NMR}\left(270 \mathrm{MHz}, \mathrm{CDCl}_{3}\right): \delta 9.02(\mathrm{~s}, 2 \mathrm{H}, \mathrm{CH}=\mathrm{N})$, $\delta 8.09 \sim 8.12(\mathrm{~d}, J=8.1 \mathrm{~Hz}, 2 \mathrm{H}$, aromatic), $\delta 6.31 \sim 6.35$ (dd, $J=7.1$ and $2.7 \mathrm{~Hz}, 2 \mathrm{H}$, aromatic), $\delta 6.03 \sim 6.04$ (d, $J=2.7 \mathrm{~Hz}, 2 \mathrm{H}$, aromatic), $\delta 4.01 \sim 4.05$ (t, $J=5.4 \mathrm{~Hz}, 4 \mathrm{H}, \mathrm{O}-\mathrm{Pen}$ ), $\delta 3.09$ (s, $12 \mathrm{H}, \mathrm{NMe}_{2}$ ), $\delta 1.81 \sim 1.90$ (m, 4H, O-Pen), $\delta 1.37 \sim 1.56$ (m, 8H, O-Pen), $\delta 0.93 \sim 0.97$ (t, $J=5.4$ Hz, 6H, O-Pen); IR (KBr): 2202 $(\mathrm{C} \equiv \mathrm{N}) \mathrm{cm}^{-1}, 1616(\mathrm{CH}=\mathrm{N}) \mathrm{cm}^{-1} .7 \mathrm{~b}$, Yield: $50.6 \%(0.17 \mathrm{~g})$; M.p. $210-211{ }^{\circ} \mathrm{C}$. Anal. Calcd for $\mathrm{C}_{42} \mathrm{H}_{62} \mathrm{~N}_{6} \mathrm{O}_{2}: \mathrm{C}, 73.86 ; \mathrm{H}, 9.15 ; \mathrm{N}, 12.30$. Found: $\mathrm{C}, 73.41 ; \mathrm{H}, 9.46 ; \mathrm{N}, 12.37$. ${ }^{1} \mathrm{H}$ NMR $(300 \mathrm{MHz}$, $\left.\mathrm{CDCl}_{3}\right): \delta 9.05(\mathrm{~s}, 2 \mathrm{H}, \mathrm{CH}=\mathrm{N}), \delta 8.11 \sim 8.14(\mathrm{~d}, J=9.0 \mathrm{~Hz}, 2 \mathrm{H}$, aromatic), $\delta 6.34 \sim 6.37(\mathrm{~d}, J=9.0 \mathrm{~Hz}, 2 \mathrm{H}$, aromatic), $\delta 6.04 \sim 6.05$ (d, $J=2.4 \mathrm{~Hz}, 2 \mathrm{H}$, aromatic), $\delta 4.02 \sim 4.06$ (t, $J=6.6 \mathrm{~Hz}, 4 \mathrm{H}, \mathrm{O}-\mathrm{Dec}), \delta 3.11(\mathrm{~s}, 12 \mathrm{H}$, $\mathrm{NMe}_{2}$ ), $\delta 1.71 \sim 1.81$ (m, 4H, O-Dec), $\delta 1.20 \sim 1.42$ (m, 28H, O-Dec), $\delta 0.85 \sim 0.90$ (t, $J=6.6 \mathrm{~Hz}, 6 \mathrm{H}, \mathrm{O}-\mathrm{Dec}$ ); IR (KBr): $2204(\mathrm{C} \equiv \mathrm{N}) \mathrm{cm}^{-1}, 1618(\mathrm{CH}=\mathrm{N}) \mathrm{cm}^{-1}$. 8b, Yield: 40.1\% (0.13 g); M.p. $283-285{ }^{\circ} \mathrm{C}$. Anal. Calcd for $\mathrm{C}_{40} \mathrm{H}_{42} \mathrm{~N}_{6} \mathrm{O}_{2}$ : C, 75.21; H, 6.63; N, 13.16: Found; C, 74.77; H, 6.61; N, 13.08. ${ }^{1} \mathrm{H}$ NMR (300 $\left.\mathrm{MHz}, \mathrm{CDCl}_{3}\right): \delta 9.13(\mathrm{~s}, 2 \mathrm{H}, \mathrm{CH}=\mathrm{N}), \delta 8.12 \sim 8.16(\mathrm{~d}, J=9.0 \mathrm{~Hz}, 2 \mathrm{H}$, aromatic), $\delta 7.19 \sim 7.48(\mathrm{~m}, 10 \mathrm{H}, \mathrm{O}-$ Bz), $\delta 6.37$ (s, 2H, aromatic), $\delta 6.06$ (s, 2H, aromatic), $\delta 5.10 \sim 5.22$ (s, 4H, O-Bz), $\delta 3.32 \sim 3.39$ (q, J=6.0 Hz, 8H, Net $\left.{ }_{2}\right), \delta 1.06 \sim 1.20\left(\mathrm{~m}, 12 \mathrm{H}, \mathrm{Net}_{2}\right)$; IR $(\mathrm{KBr}): 2203(\mathrm{C} \equiv \mathrm{N}) \mathrm{cm}^{-1}, 1612(\mathrm{CH}=\mathrm{N}) \mathrm{cm}^{-1}$. 9b, Yield: 53.5\% 
(0.16 g); M.p. 310-312 ${ }^{\circ}$ C. Anal. Calcd for $\mathrm{C}_{38} \mathrm{H}_{38} \mathrm{~N}_{6} \mathrm{O}_{2}$ : C, 74.73; H, 6.27; N, 13.76: Found; C, 74.31; H, 6.24; N, 13.65. ${ }^{1} \mathrm{H}$ NMR $\left(300 \mathrm{MHz}, \mathrm{CDCl}_{3}\right): \delta 8.98(\mathrm{~s}, 2 \mathrm{H}, \mathrm{CH}=\mathrm{N}), \delta 8.19 \sim 8.22(\mathrm{~d}, J=9.0 \mathrm{~Hz}, 2 \mathrm{H}$, aromatic), $\delta 7.33 \sim 7.40(\mathrm{~m}, 4 \mathrm{H}, \mathrm{O}-\mathrm{Ph}), \delta 7.04 \sim 7.16(\mathrm{~m}, 6 \mathrm{H}, \mathrm{O}-\mathrm{Ph}), \delta 6.53 \sim 6.56(\mathrm{~d}, J=8.8 \mathrm{~Hz}, 2 \mathrm{H}$, aromatic), $\delta 6.07\left(\mathrm{~s}, 2 \mathrm{H}\right.$, aromatic), $\delta 3.28 \sim 3.35\left(\mathrm{q}, J=7.1 \mathrm{~Hz}, 8 \mathrm{H}, \mathrm{NEt}_{2}\right), \delta 1.10 \sim 1.15(\mathrm{t}, J=7.1 \mathrm{~Hz}, 12 \mathrm{H}$, $\left.\mathrm{NEt}_{2}\right)$; IR (KBr): $2208(\mathrm{C} \equiv \mathrm{N}) \mathrm{cm}^{-1}, 1613(\mathrm{CH}=\mathrm{N}) \mathrm{cm}^{-1} . \mathbf{1 0 b}$, Yield: 72.2\% (0.18 g); M.p. $285-287^{\circ} \mathrm{C}$. Anal. Calcd for $\mathrm{C}_{28} \mathrm{H}_{34} \mathrm{~N}_{6} \mathrm{O}_{2}$ : C, 69.11; H, 7.04; N, 17.27: Found; C, 69.08; H, 7.07; N, 17.26. ${ }^{1} \mathrm{H}$ NMR $\left(270 \mathrm{MHz}, \mathrm{CDCl}_{3}\right): \delta 8.99(\mathrm{~s}, 2 \mathrm{H}, \mathrm{CH}=\mathrm{N}), \delta 8.10 \sim 8.13(\mathrm{~d}, J=9.2 \mathrm{~Hz}, 2 \mathrm{H}$, aromatic), $\delta 6.32 \sim 6.36(\mathrm{dd}, J=$ 7.0 and $2.2 \mathrm{~Hz}, 2 \mathrm{H}$, aromatic), $\delta 6.03 \sim 6.04(\mathrm{~d}, J=2.2 \mathrm{~Hz}, 2 \mathrm{H}$, aromatic), $\delta 3.88$ (s, 6H, O-Me), $\delta 3.41 \sim 3.49$ (q, $\left.J=7.0 \mathrm{~Hz}, 8 \mathrm{H}, \mathrm{NEt}_{2}\right), \delta 1.21 \sim 1.26\left(\mathrm{t}, J=7.3 \mathrm{~Hz}, 12 \mathrm{H}, \mathrm{NEt}_{2}\right) ; \mathrm{IR}(\mathrm{KBr}): 2201(\mathrm{C} \equiv \mathrm{N}) \mathrm{cm}^{-1}, 1610$ $(\mathrm{CH}=\mathrm{N}) \mathrm{cm}^{-1}$. 11b, Yield: $71.7 \%(0.18 \mathrm{~g})$; M.p. $258-259{ }^{\circ} \mathrm{C}$. Anal. Calcd for $\mathrm{C}_{30} \mathrm{H}_{38} \mathrm{~N}_{6} \mathrm{O}_{2}: \mathrm{C}, 70.01 ; \mathrm{H}$, 7.44; N, 16.33: Found; C, 69.78; H, 7.44; N, 16.27. ${ }^{1} \mathrm{H}$ NMR (300 MHz, $\left.\mathrm{CDCl}_{3}\right): \delta 9.01(\mathrm{~s}, 2 \mathrm{H}, \mathrm{CH}=\mathrm{N})$, $\delta 8.10 \sim 8.13(\mathrm{~d}, J=9.0 \mathrm{~Hz}, 2 \mathrm{H}$, aromatic), $\delta 6.33 \sim 6.36(\mathrm{dd}, J=9.0$ and $2.1 \mathrm{~Hz}, 2 \mathrm{H}$, aromatic), $\delta 6.03 \sim 6.04$ (d, $J=3.0 \mathrm{~Hz}, 2 \mathrm{H}$, aromatic), $\delta 4.08 \sim 4.15(\mathrm{dd}, J=9.0$ and $6.0 \mathrm{~Hz}, 4 \mathrm{H}, \mathrm{O}-\mathrm{Et}), \delta 3.40 \sim 3.47(\mathrm{q}, J=6.0 \mathrm{~Hz}$, $\left.8 \mathrm{H}, \mathrm{NEt}_{2}\right), \delta 1.47 \sim 1.51(\mathrm{t}, J=6.0 \mathrm{~Hz}, 6 \mathrm{H}, \mathrm{O}-\mathrm{Et}), \delta 1.21 \sim 1.25\left(\mathrm{t}, J=6.0 \mathrm{~Hz}, 12 \mathrm{H}, \mathrm{NEt}_{2}\right)$; IR $(\mathrm{KBr}): 2203$ $(\mathrm{C} \equiv \mathrm{N}) \mathrm{cm}^{-1}, 1609(\mathrm{CH}=\mathrm{N}) \mathrm{cm}^{-1}$. 12b, Yield: 71.8\% (0.19 g); M.p. 302-304 ${ }^{\circ} \mathrm{C}$. Anal. Calcd for $\mathrm{C}_{32} \mathrm{H}_{42} \mathrm{~N}_{6} \mathrm{O}_{2}$ : C, 70.82; H, 7.80; N, 15.49: Found; C, 70.02; H, 7.72; N, 15.12. ${ }^{1} \mathrm{H}$ NMR (300 MHz, $\left.\mathrm{CDCl}_{3}\right): \delta 9.02(\mathrm{~s}, 2 \mathrm{H}, \mathrm{CH}=\mathrm{N}), \delta 8.09 \sim 8.12(\mathrm{~d}, J=9.0 \mathrm{~Hz}, 2 \mathrm{H}$, aromatic), $\delta 6.32 \sim 6.36(\mathrm{dd}, J=7.2$ and 2.1 $\mathrm{Hz}, 2 \mathrm{H}$, aromatic), $\delta 6.03(\mathrm{~d}, J=2.3 \mathrm{~Hz}, 2 \mathrm{H}$, aromatic), $\delta 3.97 \sim 4.02(\mathrm{t}, J=6.5 \mathrm{~Hz}, 4 \mathrm{H}, \mathrm{O}-\mathrm{Pr}), \delta 3.40 \sim 3.47$ (q, $\left.J=7.2 \mathrm{~Hz}, 8 \mathrm{H}, \mathrm{NEt}_{2}\right), \delta 1.86 \sim 1.93(\mathrm{~m}, 4 \mathrm{H}, \mathrm{O}-\mathrm{Pr}), \delta 1.20 \sim 1.25\left(\mathrm{t}, J=7.1 \mathrm{~Hz}, 12 \mathrm{H}, \mathrm{NEt}_{2}\right), \delta 1.07 \sim 1.12(\mathrm{t}$, $J=7.3 \mathrm{~Hz}, 6 \mathrm{H}, \mathrm{O}-\mathrm{Pr})$; IR (KBr): $2201(\mathrm{C} \equiv \mathrm{N}) \mathrm{cm}^{-1}, 1612(\mathrm{CH}=\mathrm{N}) \mathrm{cm}^{-1}$. 13b, Yield: 60.8\% (0.18 g); M.p. 270-271 ${ }^{\circ} \mathrm{C}$. Anal. Calcd for $\mathrm{C}_{36} \mathrm{H}_{50} \mathrm{~N}_{6} \mathrm{O}_{2}$ : C, 72.21; H, 8.42; N, 14.03: Found; C, 71.92; H, 8.41; N, 13.94 . ${ }^{1} \mathrm{H}$ NMR $\left(270 \mathrm{MHz}, \mathrm{CDCl}_{3}\right): \delta 9.02(\mathrm{~s}, 2 \mathrm{H}, \mathrm{CH}=\mathrm{N}), \delta 8.09 \sim 8.13(\mathrm{~d}, J=9.5 \mathrm{~Hz}, 2 \mathrm{H}$, aromatic), $\delta 6.31 \sim 6.35$ (dd, $J=5.4$ and $2.7 \mathrm{~Hz}, 2 \mathrm{H}$, aromatic), $\delta 6.03 \sim 6.04(\mathrm{~d}, J=2.7 \mathrm{~Hz}, 2 \mathrm{H}$, aromatic), $\delta 4.01 \sim 4.05(\mathrm{t}, J=5.4$ Hz, 4H, O-Pen), $83.40 \sim 3.47$ (dd, $J=8.1$ and $5.4 \mathrm{~Hz}, 8 \mathrm{H}, \mathrm{NEt}_{2}$ ), $\delta 1.56 \sim 1.90$ (m, 4H, O-Pen), $\delta 1.37 \sim 1.56$ 
(m, 8H, O-Pen), $\delta 1.20 \sim 1.25\left(\mathrm{t}, J=5.4 \mathrm{~Hz}, 12 \mathrm{H}, \mathrm{NEt}_{2}\right), \delta 0.93 \sim 0.98$ (t, $\left.J=5.4 \mathrm{~Hz}, 6 \mathrm{H}, \mathrm{O}-\mathrm{Pen}\right)$; IR $(\mathrm{KBr})$ : $2201(\mathrm{C} \equiv \mathrm{N}) \mathrm{cm}^{-1}, 1614(\mathrm{CH}=\mathrm{N}) \mathrm{cm}^{-1}$. 14b, Yield: 58.9\% (0.22 g); M.p. 192-194 ${ }^{\circ} \mathrm{C}$. Anal. Calcd for $\mathrm{C}_{46} \mathrm{H}_{70} \mathrm{~N}_{6} \mathrm{O}_{2}:$ C, 74.75; H, 9.55; N, 11.37: Found; C, 74.88; H, 9.59; N, 11.37. ${ }^{1} \mathrm{H}$ NMR $(270 \mathrm{MHz}$, $\left.\mathrm{CDCl}_{3}\right): \delta 9.01(\mathrm{~s}, 2 \mathrm{H}, \mathrm{CH}=\mathrm{N}), \delta 8.09 \sim 8.13(\mathrm{~d}, J=8.1 \mathrm{~Hz}, 2 \mathrm{H}$, aromatic), $\delta 6.32 \sim 6.36(\mathrm{dd}, J=5.4$ and 2.7 $\mathrm{Hz}, 2 \mathrm{H}$, aromatic), $\delta 6.03 \sim 6.04(\mathrm{~d}, J=2.7 \mathrm{~Hz}, 2 \mathrm{H}$, aromatic), $\delta 4.00 \sim 4.05(\mathrm{t}, J=5.4 \mathrm{~Hz}, 4 \mathrm{H}, \mathrm{O}-\mathrm{Dec})$, $\delta 3.40 \sim 3.47\left(\mathrm{q}, J=5.4 \mathrm{~Hz}, 8 \mathrm{H}, \mathrm{NEt}_{2}\right), \delta 1.81 \sim 1.91$ (m, 4H, O-Dec), $\delta 1.20 \sim 1.52\left(\mathrm{~m}, 40 \mathrm{H}, \mathrm{O}-\mathrm{Dec}_{\text {and }} \mathrm{NEt}_{2}\right.$ ), $\delta 0.85 \sim 0.90(\mathrm{t}, J=5.4 \mathrm{~Hz}, 6 \mathrm{H}, \mathrm{O}-\mathrm{Dec})$; IR $(\mathrm{KBr}): 2207(\mathrm{C} \equiv \mathrm{N}) \mathrm{cm}^{-1}, 1609(\mathrm{CH}=\mathrm{N}) \mathrm{cm}^{-1}$.

\section{Results and Discussion}

Several reports on the crystal structure of bisazomethine dyes have revealed that their crystal structure is characterized by a two-dimensional (2-D) molecular stacking layer in which molecules are $\pi$ $\pi$ stacked in a staircase arrangement with small slip angles along the long molecular axis [26, 28]. The 2D layers are aligned along the short molecular axis such that they form a crystal structure. The observed 2-D molecular stacking layer feature indicated that the bisazomethine dye system has the potential to form J-aggregates and, indeed, DE2 was found to form J-aggregates in vapour-deposited films [31], although those films were composed of J-aggregates and a single crystalline phase.

With respect to the molecular arrangement along the short molecular axis, the cyano group, the substituents on the phenyl ring, and the alkyl substituents on the amino group are related by the spacing between the adjacent 2-D stacking layers. This spacing between the neighbouring layers is regarded as one of the important parameters for obtaining pure J-aggregate solid films, because J-aggregates are considered to have a low-dimensional electronic state. Among the different substituents, phenyl ring substitution was examined in this study. 4-(Dimethylamino)- and 4-(diethylamino)-2alkoxybenzaldehydes 1a-14a were reacted with diaminomaleonitrile to yield bisazomethine dyes $\mathbf{1 b}-\mathbf{1 4 b}$, 
respectively, to obtain J-aggregates in the solid state. The active amino groups of diaminomaleonitrile can readily react with the aldehyde group to afford azomethine linkages. All the prepared novel bisazomethine dyes were obtained in yields of $40-78 \%$.

[Scheme 1]

The UV-Vis absorption and fluorescence spectra of $\mathbf{1 b}-\mathbf{7 b}$ and $\mathbf{8 b}-\mathbf{1 4 b}$ in chloroform are shown in Figs. 1 (a) and (b), respectively. All the synthesized bisazomethine dyes show strong absorption around $550 \mathrm{~nm}$ with a very large molar extinction coefficient of about 120000 to 150000 , which confirms the symmetrical strong intramolecular charge-transfer chromophoric system of the bisazomethine dye molecule [39]. All the absorption bands have a small shoulder around $520 \mathrm{~nm}$, and no significant difference was observed in their spectral shape. Dyes $\mathbf{1 b}-\mathbf{7 b}$ exhibit a sharp fluorescent band around 595 nm. Dyes 8b-14b also show a sharp fluorescent band but the spectral region is slightly shifted to the long-wavelength region. Thus, these derivatives can be used as a fluorescent material for a red emitter, although their quantum efficiency is very low. All the dyes showed a Stokes shift of about $45 \mathrm{~nm}$. These experimental data clearly indicate that the substitution in the amino groups as well as in the alkoxy groups on the phenyl ring exerts no considerable influence on the electronic states in the visible region.

\section{[Figure 1]}

To gain further insight into the electronic states of these bisazomethine dyes, their molecular geometry and absorption characteristics were computed. The optimized molecular geometry for $\mathbf{1 b}$ and 8b is illustrated in Figs. 2 (a) and (b), respectively. The molecule has an almost planar $\pi$-conjugation from the terminal amino group to the central dicyanoethylene moiety. This molecular conformation implies that a strong intramolecular charge-transfer exists in this chromophoric system upon excitation. The absorption corresponding to the visible absorption was estimated on the basis of configuration interactions. The calculation result is listed in Table 1. A strong absorption was observed for all dyes whose oscillator strengths ranged from 1.9 to 2.0. We found no significant difference in the calculated 
absorption energy. This result is in good agreement with the absorption characteristics shown in Fig. 1. The major component of this transition is the HOMO-LUMO transition. The HOMO was found to consist of amino nitrogen atoms including phenyl carbons and the central ethylene moiety. The LUMO is mainly distributed over the azomethine and dicyanoethylene moieties. The transition dipole was found to be oriented along the long molecular axis parallel to the direction from the amino nitrogen to its conjugated cyano nitrogen. This reflects exactly the conjugation of $\pi$-electrons between the terminal amino donors and the central cyano groups. A cross-conjugation through the central ethylene moiety is also suggested and this is considered the reason for the very large oscillator strength of these bisazomethine dyes.

[Figure 2]

[Table 1]

These experimental results clearly indicate that for all the prepared bisazomethine dyes in a solution, there is little effect of the substituents on the optical properties of the dyes in the visible region. This, of course, is attributed to the small electronic effect of the substituents on the $\pi$-conjugation of the chromophoric system. These novel bisazomethine dyes, however, exhibit a significant spectral change in solid films. Figs. 3 and 4 show the absorption and fluorescent spectra of $\mathbf{1 b}-\mathbf{7 b}$ and $\mathbf{8 b}-\mathbf{1 4 b}$, respectively, in a vapour-deposited film. These spectral characteristics are summarized in Table 2, along with the spectral characteristics in a chloroform solution. The spectral shift between the solution and the films is also listed.

[Figure 3]

[Figure 4]

[Table 2]

The shape of the absorption bands of $\mathbf{3 b}$ and $\mathbf{1 0 b}$ is noteworthy. They are characterized by a broad band over the visible region with a sharp peak around $650 \mathrm{~nm}$. The shift between the peaks of the solution spectra and film spectra is $103 \mathrm{~nm}$ in the case of both dyes. This spectral change is quite similar to that of 
DE2 [31], which shows a sharp peak - that was assigned to J-aggregates - in the long-wavelength region in vapour-deposited films. Thus, J-aggregates are considered to form in these films; to obtain pure Jaggregate films, the preparation conditions of the films will have to be examined. In the case of other dyes, both band broadening and bathochromic spectral shifts in the absorption spectra were observed. The degree of bathochromic shift is dependent on the alkyl chain length of the alkoxy substituents on the phenyl rings. The methoxy group (3b and $\mathbf{1 0 b}$ ) showed the largest shift in absorption, and the spectral shift decreased with increasing chain length in both series. The decyloxy group (7b and $\mathbf{1 4 b}$ ) showed the shortest spectral shift. The alkoxy substituents were introduced to modulate the spacing between the 2-D stacking molecular layers. They also have an influence on the geometry of the molecular stacking in the 2-D layer. This observation may thus reflect some structural variety in molecular arrangement in the solid state. However, the dye films showed a simple fluorescence band around 620-690 nm in the solid state. The shape of the peak was slightly different from that in the solution. There were no significant differences in the spectral shifts for the dyes except for $\mathbf{1 b}$ and $\mathbf{8 b}$. Among all the dyes, $\mathbf{1 b}$ and $\mathbf{8 b}$ showed quite an unusual absorption band with two distinct absorption maxima around $450 \mathrm{~nm}$ and $600 \mathrm{~nm}$. A theory to explain this phenomenon was proposed: during vacuum deposition, both dyes are thermally decomposed to give mono-substituted azomethine dyes, which show the hypsochromic absorption band. This theory was experimentally confirmed by preparing the corresponding monoazomethine dyes and their vapour-deposited films. These bisazomethine dyes showed significant spectral difference in the solid state, despite their molecules having almost similar electronic states. Therefore, the spectral shift is correlated with the solid-state structure; the spectral shift is considered to depend on the alkoxy substituents on the phenyl ring.

The absorption bands for $\mathbf{3 b}, \mathbf{9 b}, \mathbf{1 0 b}$, and $\mathbf{1 1 b}$ in vapour-deposited films are illustrated in Fig. 5, along with their absorption spectra in chloroform solution. As mentioned above, the shape of the spectra of $3 \mathbf{b}$ and 10b was very similar to that of DE2 in vapour-deposited films and their peaks around $650 \mathrm{~nm}$ 
can be ascribed to J-aggregate formation. In addition to these two dyes, $\mathbf{9 b}$ and $\mathbf{1 1 b}$ exhibited a tendency to form J-aggregates in vapour-deposited films. In their film absorption bands, the absorption spectrum increases very sharply around the absorption edge in the long-wavelength region. In the solution spectrum, the bisazomethine dyes generally exhibit a well-defined peak as well as a relatively small Stokes shift of about $1400 \mathrm{~cm}^{-1}$. This suggests that the major absorption peak can be assigned to the $0-0$ transition. In the case of $9 \mathrm{~b}$ and 11b, the rise in the absorption edge in the longer wavelength region was steeper in the case of the film absorption spectra than in the case of the solution spectra. This implies that a pure electronic transition in the longer-wavelength region, namely, J-aggregate absorption, is incorporated in the film absorption spectra. Among all dyes, four dyes-3b, $\mathbf{9 b}, \mathbf{1 0 b}$, and $\mathbf{1 1 b}$-were considered to form Jaggregates in vapour-deposited films, on the basis of the shape of the absorption spectrum and absorption edge.

\section{[Figure 5]}

For an exact interpretation of these spectral changes and to achieve our final goal of obtaining pure Jaggregate solid films, the preparation conditions of the films will have to be examined and the optical properties of the dyes will have to be characterized on the basis of the solid-state structure. Crystal structure analysis of these dyes is thus very important and the results will be reported elsewhere.

\section{Conclusions}

In an attempt to prepare pure J-aggregate solid films, we investigated the effect of phenyl ring substitution in bisazomethine chromophoric systems, synthesized using diaminomaleonitrile and aminobenzaldehydes, with alkoxy groups. In chloroform solution, all the synthesized bisazomethine dyes exhibited almost similar optical properties, namely, strong absorption around $550 \mathrm{~nm}$ and weak red fluorescence around $590 \mathrm{~nm}$. Molecular orbital calculations confirmed the strong intramolecular charge- 
transfer property of the synthesized dyes and the calculation result was in good agreement with their solution properties. In the case of vapour-deposited films, the absorption spectra of the dyes showed a variety of spectral changes in the visible region. Four of the synthesized derivatives were found to form Jaggregates in vapour-deposited films, as determined from the shape of the spectrum and the absorption edge. Further investigation of the observed spectral changes, which involves crystal structure analysis of all the dyes, is underway. In addition, to obtain pure J-aggregate solid films, the various preparation conditions of the films and other combinations of substituents will be examined.

\section{Acknowledgement}

This research was financially supported by the Japan Society for the Promotion of Science (JSPS) and the Korea Science and Engineering Foundation (KOSEF) under the Japan-Korea Basic Scientific Cooperation Program as well as the Sasagawa Scientific Research Grant from the Japan Science Society.

\section{References}

[1] Sung HH, Kim IH, Byun KN, Yoo HS, Characterization of new red dopants for red color OLED, Current Applied Physics 2005;5(4):345-347.

[2] Bubniene G, Malinauskas T, Daskeviciene M, Jankauskas V, Getautis V, Easily functionalizable carbazole based building blocks with extended conjugated systems for optoelectronic applications, Tetrahedron 2010;66(17):3199-3206.

[3] Shang H, Fan H, Shi Q, Li S, Li Y, Zhan X, Solution processable D-A-D molecules based on triphenylamine for efficient organic solar cells, Solar Energy Materials and Solar Cells 2010;94(3):457-464.

[4] Wong WY, Ho CL, Functional metallophosphors for effective charge carrier injection/transport: New 
robust OLED materials with emerging applications, Journal of Materials Chemistry 2009;19:44574482.

[5] Walzer K, Maenning B, Preiffer M, Leo K, Highly efficient organic devices based on electrically doped transport layers, Chemical Reviews 2007;107:1233-1271.

[6] Zaumseil J, Sirringhaus H, Electron and ambipolar transport in organic field-effect transistors, Chemical Reviews 2007;107:1296-1323.

[7] Dimitrakopoulos CD, Malenfant PRL, Organic thin film transistors for large area electronics, Advanced Materials 2002;14(2):99-117.

[8] Armstrong NR, Wang W, Alloway DM, Placencia D, Ratcliff E, Brumbach M, Organic/organic' heterojunctions: Organic light emitting diodes and organic photovoltaic devices, Macromolecular Rapid Communications 2009;30:717-731.

[9] Hadipour A, Boer BD, Blom PWM, Organic tandem and multi-junction solar cells, Advanced Functional Materials 2008;18(2):169-181.

[10] Bricaud Q, Cravino A, Leriche P, Roncali J, Terthiophene-cyanovinylene $\pi$-conjugated polymers as donor material for organic solar cells, Synthetic Metals 2009;159(23-24):2534-2538.

[11] Lee HN, Lee YG, Ko IH, Hwang EC, Kang SK, Organic passivation layers for pentacene organic thin-film transistors, Current Applied Physics 2008;8(5):626-630.

[12] Anthony JE, Functionalized acences and heteroacences for organic electronics, Chemical Reviews 2006;106(12):5028-5048.

[13] Xue C, Luo FT, Synthesis of oligo(p-phenylene-vinylene-thienylene)s as potential red light-emitting materials, Tetrahedron 2003;59(28):5193-5198.

[14] Fu HY, Zhan YQ, Xu JX, Hou XY, Xiao F, Red fluorescent materials based on naphthylamine for non-doping OLEDs, Optical Materials 2006;29(2-3):348-354.

[15] Matsumoto M, J-aggregate formation of dyes in Langmuir-Blodgett films, Journal of 
Photochemistry and Photobiology A: Chemistry 2003;158(2-3):199-203.

[16] Ikegami K, Lan M, Vibrational modes of merocyanine dyes softened upon J-aggregation of the dyes in their Langmuir-Blodgett films, Colloids and Surfaces A: Physicochemical and Engineering Aspects 2005;257-258:143-148.

[17] Kato N, Yuasa K, Araki T, Hirosawa I, Sato M, Ikeda N, Iimura KI, Uesu U, Determination of a merocyanine J-aggregate structure and the significant contribution of the electric dipole interaction to the exciton band wavelength, Physical Review Letters 2005;94(13):136404.1-136404.4.

[18] Wang M, Silva GL, Armitage BA, DNA-templated formation of a helical cyanine dye J-aggregate, Journal of the American Chemical Society 2000;122(41):9977-9986.

[19] Kuroda SI, J-aggregation and its characterization in Langmuir-Blodgett films of merocyanine dyes, Advances in Colloid and Interface Science 2004;111(3):181-209.

[20] Smith DL, Structure of dyes and dye aggregates-evidence from crystal structure analyses, Photography Science and Engineering 1974;18:309-322.

[21] Kobayashi T, J-aggregates, World Scientific Publishing, Singapore, 1996.

[22] Möbius D, Scheibe aggregates, Advanced Materials 1995;7(5):437-444.

[23] Davydov AS, Theory of Molecular Excitons, McGraw-Hill, New York, 1962.

[24] Scheibe G, Die stereoisomerie organischer farbstoffe und ihr zusammenhang mit konstitution und eigenschaften reversible polymerer farbstoffe, Angewandte Chemie 1939;52:631-637.

[25] Czikkely V, Försterling HD, Kuhn H, Light absorption and structures of aggregates of dye molecules, Chemical Physics Letters 1970;6(1):11-14.

[26] Matsumoto S, Shirai K, Kobayashi K, Wada T, Shiro M, J-aggregate structures in crystals of three bisazomethine dyes, Zeitschrif fur Kristallographie 2004;219;239-243.

[27] Kim SH, Matsumoto S, Electrochromism and X-ray crystal structure of a new azomethine dye derived from diaminomaleonitrile, Dyes and Pigments 2007;72(3):406-408. 
[28] Son YA, Matsumoto S, Han EM, Wang S, Kim SH, Electro-optical properties and X-ray crystal structure of a new bisazomethine dye, Molecular Crystals and Liquid Crystals 2008;492:46/[410]$55 /[419]$.

[29] Nakatsu K, Yoshioka H, Nishigaki S, Structural models for low-dimensional photographic dye aggregates as revealed by X-ray crystallographic studies, Nihon Shashin Gkkai-shi (in Japanese) 1983;46:89-98.

[30] Matsumoto S, Satoh M, Kobayashi T, Aoyama T, Wada T, Morphological characteristics of Jaggregate films of bisazomethine dyes, Transactions of the Materials Research Society of Japan $2005 ; 30(2): 345-348$.

[31] Matsumoto S, Kobayashi T, Aoyama T, Wada T, J-aggregates in vapor deposited films of a bisazomethine dye, Chemical Communications 2003;15:1910-1911.

[32] Kobayashi T, Matsumoto S, Tanaka T, Kunugita H, Ema K, Aoyama T, Wada T, Photoluminescence properties of vapor deposited films of a bisazomethine dye, Physical Chemistry Chemical Physics $2005 ; 7: 1726-1731$

[33] Hosokai T, Aoyama T, Kobayashi T, Nakao A, Matsumoto S, Photovoltic properties of bisazomethine dye thin films, Chemical Physics Letters 2010;487:77-80.

[34] Dewar MJS, Zoebish EJ, Healy EF, Stewart JJP, Development and use of quantum mechanical molecular models. 76. AM1: A new general purpose quantum mechanical molecular model, Journal of the American Chemical Society 1985;107(13):3902-3909.

[35] Pople JA, Beveridge DL, Dobosh PA, Approximate self-consistent molecular-orbital theory.V. Intermediate neglect of differential overlap, The Journal of Chemical Physics 1967;47(6):2026-2033.

[36] Kotzian M, Rosch N, Scroder H, Zerner MC, Optical spectra of transition-metal carbonyls: Chromium hexacarbonyl, iron pentacarbonyl, and nickel tetracarbonyl, Journal of the American Chemical Society 1989;111(20):7687-7696. 
[37] Fujitsu Limited, Computer aided chemical modeling package, Tokyo, Japan, 2001.

[38] Shirai K, Matsuoka M, Fukunishi K, New syntheses and solid state fluorescence of azomethine dyes derived from diaminomaleonitrile and 2,5-diamino-3,6-dicyanopyrazine, Dyes and Pigments 2000;47(1-2):107-115.

[39] Kim SH, Yoon SH, Kim SH, Han EM, Red electroluminescent azomethine dyes derived from diaminomaleonitrile, Dyes and Pigments 2005;64(1):45-48.

[40] Wang YJ, Sheu HS, Lai CK, New star-shaped triarylamines: synthesis, mesomorphic behavior, and photophysical properties Tetrahedron 2007;63(7):1695-1705.

[41] Jung BJ, Lee JI, Chu HY, Do LM, A new family of bis-DCM based dopants for red OLEDs, Journal of Materials Chemistry 2005;15:2470-2475.

[42] Kwon OP, Rezzonico D, Kwon SJ, Jazbinsek M, Günter P, New nonlinear optical polyamides: Influence of binding mode of side-chains and rigidity of main-chains on temporal stability, European Polymer Journal 2008;44(7):2219-2224.

[43] Gulajski L, Michrowska A, Bujok R, Grela K, New tunable catalysts for olefin metathesis: Controlling the initiation through electronic factors, Journal of Molecular Catalysis A: Chemical 2006;254(1-2):118-123.

[44] Shen G, Lv X, Qian W, Bao W, $\mathrm{Cu}_{2} \mathrm{O}$-catalyzed Ullmann-type reaction of vinyl bromides with imidazole and benzimidazole, Tetrahedron Letter 2008;49(29-30):4556-4559.

[45] Kidwai M, Mishra NK, Bansal V, Kumar A, Mozumdar S, Cu-nanoparticle catalyzed O-arylation of phenols with aryl halides via Ullmann coupling, Tetrahedron Letter 2007;48(50):8883-8887.

[46] Xi Z, Liu F, Zhou Y, Chen W, CuI/L (L=pyridine-functionalized 1,3-diketones) catalyzed C-N coupling reactions of aryl halides with NH-containing heterocycles, Tetrahedron 2008;64(19):42544259. 


\section{Captions}

Scheme 1

\section{List of Figures}

Fig. 1. Absorption and fluorescence spectra of $\mathbf{1 b}-\mathbf{7 b}$ (a) and $\mathbf{8 b}-\mathbf{1 4 b}(\mathrm{b})$ in chloroform solution. The concentration of dyes for fluorescence is ca. $1.0 \times 10^{-5} \mathrm{M}$.

Fig. 2. Optimized molecular geometry for $\mathbf{1 b}(\mathrm{a})$ and $\mathbf{8 b}(\mathrm{b})$.

Fig. 3. Absorption (a) and fluorescence (b) spectra of $\mathbf{1 b}-\mathbf{7 b}$ in vapour-deposited films.

Fig. 4. Absorption (a) and fluorescence (b) spectra of $\mathbf{8 b}-\mathbf{1 4 b}$ in vapour-deposited films.

Fig. 5. Absorption spectra of $\mathbf{3 b}, \mathbf{9 b}, \mathbf{1 0 b}$ and $\mathbf{1 1 b}$ in vapour-deposited films, as well as the spectra in chloroform.

\section{List of Tables}

Table 1. Calculated absorption parameters for the optimized molecular geometry.

Table 2. Absorption and fluorescence characteristics for $\mathbf{1 b} \mathbf{- 1 4 \mathbf { b }}$ in solution and in solid films. 